\title{
Burnout on the frontline: the impact of COVID-19 on emergency department staff wellbeing
}

\author{
Oisín Sheehan ${ }^{1}$ (D) Micheal Sheehan ${ }^{1} \cdot$ Raluca lleana Rau $^{1} \cdot$ Iomhar O. Sullivan ${ }^{1} \cdot$ Geraldine McMahon $^{1} \cdot$ Ann Payne $^{1}$
}

Received: 21 May 2021 / Accepted: 26 September 2021 / Published online: 9 October 2021

(c) The Author(s), under exclusive licence to Royal Academy of Medicine in Ireland 2021

\begin{abstract}
Background Burnout is a syndrome resulting from chronic workplace stress that has not been successfully managed. Healthcare professionals are particularly susceptible to this occupational phenomenon. There is limited literature currently published addressing burnout in the context of the Irish frontline workers during the COVID-19 pandemic.

Objectives Our study aims to determine the rate and degree of burnout present in the emergency department (ED) staff at St. James hospital Dublin (SJH) and at Cork University Hospital (CUH), in the context of the COVID-19 pandemic.

Design A cross-sectional study was performed on a convenience sample of medical staff and healthcare staff in the ED at Cork University Hospital and St. James Hospital Dublin, two major University Hospital EDs. Burnout was measured using the Oldenburg burnout inventory (OLBI) a standardised 16-question instrument. Participants also provided demographic data and answered several questions relating to the pandemic.

Participants Ninety-nine participants across two departments responded (a response rate of 30\%). All ED cohorts were represented.

Results Burnout was identified in almost three quarters of respondents (74\%). The mean burnout scores were 2.42 (OLBI cut-off 2.18). There was mean disengagement level of 2.25 and a mean exhaustion level of 2.59. There was no statistically significant variance between staff, by demographics (i.e. occupation, years working in the ED, age, or gender). There was no significant difference in burnout rates or scores between St. James Hospital, Dublin, and Cork University Hospital, though the former tended to have a higher rate of burnout at $81 \%$ vs the $67 \%$ burnout observed in CUH.

Conclusions The COVID-19 pandemic may be contributing to the long-established problem of health care burnout. The trajectory of this disease is still unclear. Consistent, progressive measures to address staff well-being, and support frontline workers, are imperative going forward.
\end{abstract}

Keywords Burnout · COVID-19 · Emergency department staff

\section{Introduction}

Burnout can be defined as a syndrome resulting from chronic workplace stress that has not been successfully managed [1]. Over the last 10 years, burnout has become an increasingly researched and well understood phenomenon. It is characterized by energy depletion or emotional exhaustion, negativity relating to one's profession and a reduced professional efficacy [1].

Oisín Sheehan

oisintheredeemer@gmail.com

1 University College Cork, Cork, Ireland
Healthcare professionals are particularly susceptible to this occupational phenomenon. Burnout is often considered a consequence of the demanding nature of healthcare. As a result, the rate, and levels of burnout among health care professionals, is consistently high $[2,3]$.

The impact of burnout on healthcare is substantial. Burnout negatively effects staff wellbeing, it impacts the patients dealt with, and indeed the overall healthcare system [4-6]. Burnout has been linked to a wide variety of stress-related mental and physical health conditions. These range from anxiety, depressive disorders and suicidality, to physical disorders such as hypertension, stroke and MI [4-6].

Professionals who are suffering from burnout may develop a mental and emotional detachment from their coworkers, their patients and indeed their loved ones. Burnout 
also increases absenteeism and increases the rate of error in healthcare; thus, it has a negative impact on patient outcomes $[4,5]$.

There is a large degree of controversy as to what constitutes burnout and the cut-offs which should be applied. As a result of this and other factors, the rate of burnout tends to vary significantly between papers. Doctors do not Do-little: a national cross-sectional study of hospital doctors in Ireland was published in 2019 by Blanaid Hayes et al. [8]. In this paper, $29.7 \%$ of a sample of 1749 doctors' Irish doctors were found to have significant levels of burnout. There are several studies that have demonstrated that burnout rates in ED staff tend to be significantly higher than burnout rates in other departments. One major systematic review found the rate of burnout in ED physicians to be $>50 \%$ higher (60 vs $38 \%$ ) when compared to the physician population as a whole [6]. Further papers suggest this may be a conservative estimate, reporting burnout levels 3 times higher in the emergency physician population [7].

Papers addressing burnout across the ED are rare; however, one Irish paper by Chernoff et al., conducted in 2016, found that $75 \%$ of ED staff at Cork University Hospital (CUH) met the criteria for burnout. This study applied the same parameters as our study (namely the Oldenburg burnout inventory (OLBI) with a cut-off of 2.18; see below for further discussion.)

On March 11, 2020, the World Health Organization [20] declared the COVID-19 outbreak a pandemic. As of the 6th of February 2021 (the date of submission of this study), 105 million people worldwide had been infected with COVID19. There have been over 2.3 million deaths globally. In Ireland, we had seen over 200,000 cases and over 3600 deaths [19].

We are currently experiencing a period of substantial upheaval to the structure and nature of work in Ireland's EDs, and the impact of COVID-19 on hospital staff is not yet fully understood. At the time this questionnaire was distributed (December 2020), there was no curative treatment for COVID-19. There was also no distribution of vaccines. COVID-19 has a high person-to-person transmission, and the prevention of spread remains focused on PPE, hand hygiene and physical distancing of persons.

Several new COVID-era studies have been able to draw associations between identifiable features of life during a pandemic, and mental health issues for healthcare professionals [9-11].

These factors include a fear of virus exposure and occupational risk, increased work hours and a disruption of work life balance, a disruption of family life and support networks and a lack of communication associated with a rapidly evolving work environment $[9,10]$. When considering the impact of COVID 19 on health care professionals, we can also draw on studies conducted during past epidemics. One study which investigated the impact of the SARS outbreak on the mental wellbeing found a significant increase in psychological conditions throughout and in the wake of the crisis among hospital workers [11].

However, some evidence has contradicted the above prediction of negative mental health outcomes among health care practitioners. One recently published study examining burnout levels in Chinese frontline workers during the pandemic found some surprising results [12]. They discovered that there was a significantly lower level of reported burnout in doctors working directly with COVID-19 patients when compared to pre-pandemic levels. The article concluded by postulating that frontline workers had an increased engagement with work and motivation to work and that this was protective against burnout. It should also be acknowledged that hospital funding has been increased significantly, and there have been many positive changes to the emergency departments (EDs); indeed, one article in the Irish Times described the pandemic as "the best thing to happen to healthcare in 20 years" [21].

The studies reported above offer insights into the trials and triumphs associated with the pandemic. However, the challenging psychosocial environment that COVID-19 has brought, and its sequelae, remains a mystery from a research perspective. The real burden of the pandemic on healthcare staff remains to be seen.

This study will evaluate burnout levels among ED staff at two major urban hospitals during the COVID-19 pandemic. Our results will improve our understanding and may aid the early recognition of future psychosocial challenges affecting emergency departments and staff.

\section{Aims}

This study primarily aims to determine the prevalence and degree of burnout and occupational stress experienced by the ED staff of Cork University Hospital (CUH) and St. James's Hospital (SJH), Dublin. This study was carried out during the COVID-19 pandemic. We aim to measure the impact of the pandemic on staff burnout levels and put this in context regarding the existing literature. As a secondary aim, we wish to address how a variety of factors associated with the pandemic have impacted on the mental wellbeing of healthcare providers and to examine how the demographic variance of healthcare providers impacts burn-out rates and levels.

\section{Methods}

\section{Project design}

This is a cross-sectional study performed on a convenience sample of healthcare workers in the ED at Cork University Hospital (CUH) and St. James Hospital (SJH), Dublin. 
Sample. All ED staff currently working in CUH and SJH were approached in person and invited to participate in the study. Staff were recruited across a range of disciplines; nurses, physicians, radiographers, physiotherapists, phlebotomists and auxiliary staff (administrators, health care assistants, security staff and porters).

Data Collection. Information posters were displayed in the EDs of CUH and SJH (Appendix). These posters were placed 2 weeks prior to commencement of data collection and remained in the departments until the collecting process had concluded. The participants were briefed on the study in person by the clinical team leaders in each department.

A questionnaire was distributed to consenting participants by the two data collectors, one at each site. This survey was distributed at varying times of the day over a 6-week period during November and December 2020. Participants were then given a brief verbal introduction to the study as well as leaflet containing information about the study and information regarding support services, such as the Employee Assistance Program (EAP), which they could avail of if they felt impacted by occupational burnout (Appendix). Responses were anonymised and recorded either by hard copy paper surveys or on an electronic tablet which was provided by the data collector.

This project received ethical approval from the NREC COVID-19 special committee on the 4th of August 2020.

\section{Study measures}

Our project assessed burnout levels in staff by utilising the Oldenburg Burnout Inventory (OLBI) (Appendix) a validated and commonly employed questionnaire. It is considered to have validity congruent to the Maslach Burnout Inventory (MBI) which is often regarded as the gold-standard burnout tool [7, 11, 12]. The OLBI was chosen over the MBI for two reasons; it is considerably shorter than the MBI, and it has been used in the past to conduct research on CUH ED staff, thus allowing the two studies to be more comparable.

The OLBI is an instrument comprising 16 questions. The OLBI measures disengagement from work (8 questions) and exhaustion (8 questions). These two dimensions are the essential elements of burnout. Half of these questions are negatively phrased, and half are positively phrased [7, 12] thereby combatting selection biases.

Also included in the survey were additional questions relating specifically to the COVID-19 pandemic and how employees felt resultant changes, both generally and within the emergency department, had impacted on their wellbeing. This was to further elucidate the influence of the pandemic on staff wellbeing.

The following where considered: an increased requirement for PPE, the requirement of social distancing between staff, the decrease in the number of visitors attending with patients, the increase in pre-hospital screening, the increased media coverage of healthcare workers and resultant change in public perception of frontline workers, the decreased willingness of patients to visit ED and finally changes to the structure and layout of the ED Requirement of social distance outside of work.

Demographic data was recorded: age, their role within the emergency department, their duration of employment in the emergency department and gender. We also included three questions relating to both a history of and current experience of depression.

These where "Do you feel depressed?", "Do you have any experience of depression in the past?" and "Have you lost interest in things you previously enjoyed?".

These responses were self-reported and therefore prone to bias; however, depression has been strongly linked to burnout in many previous studies [15].

\section{Data analysis}

Data was analysed via the scoring protocol supplied with the OLBI [13].

Participants chose one of four answers for each of the 16 questions on the OLBI. They were marked 1, 2, 3 or 4 on each in accordance with the protocol [13]. The sum of each question is added, and the overall total is then divided by 16 . As such, participants scores can range from 1 (lowest score) to 4 (highest level of burnout) A score of greater than or equal to 2.18 was selected to determine the presence of burnout. While there is no absolute consensus on the cut-off and burnout is a spectrum, this score was selected as it converged with previous studies, including the study conducted in 2016 on burnout in the emergency department of CUH [14, 15].

\section{Statistical analysis}

All the analyses were performed using commercially available statistical software (Microsoft Excel and IBM SPSS statistics 26). We analysed score distribution using a histogram and Shapiro-Wilk test. We used the Pearson' chi-square test to investigate for a relationship between categorical data (burnout rates), and $t$-test to investigate for relationships between categorical and continuous data (burnout scores). 
Table 1 Demographics percentage burnout and mean scores on OLBI

\begin{tabular}{lllll}
\hline Variable & & Number & $\%$ burnout & Mean: \\
\hline Hospital & SJH & $48 / 150(30 \%)$ & $81.25 \%$ & 2.42 \\
Occupation & CUH & $51 / 176(29 \%)$ & $66.66 \%$ & 2.41 \\
Age & Nurse & $35 / 111(32 \%)$ & $77 \%$ & 2.47 \\
Years In ED & Physician & $34 / 85(40 \%)$ & $76 \%$ & 2.4 \\
Gender & Physiotherapist & $2 / 12(17 \%)$ & $50 \%$ & 2.36 \\
& Phlebotomist & $1 / 4(25 \%)$ & $100 \%$ & 2.78 \\
& Paramedic & $4 / 40(10 \%)$ & $75 \%$ & 2.61 \\
& Radiographer & $3 / 13(23 \%)$ & $0 \%$ & 1.8 \\
& Auxiliary staff & $20 / 61(33 \%)$ & $75 \%$ & 2.38 \\
& $<25$ & 8 & $25 \%$ & 2.18 \\
& 25-30 & 23 & $73.9 \%$ & 2.45 \\
& $30-40$ & 40 & $82.9 \%$ & 2.45 \\
& $40-50$ & 21 & $71.43 \%$ & 2.44 \\
& $50+$ & 7 & $71.43 \%$ & 2.32 \\
& $<1$ & 23 & $65 \%$ & 2.38 \\
& $1-5$ & 31 & $64.5 \%$ & 2.36 \\
& $5-10$ & 18 & $88.8 \%$ & 2.58 \\
& $10+$ & 27 & $81.5 \%$ & 2.43 \\
& Male & 39 & $71.8 \%$ & 2.43 \\
& Female & 60 & $73.3 \%$ & 2.42 \\
\hline
\end{tabular}

\section{Results}

This study had 99 participants in total, comprising 51 individuals employed at Cork University Hospital and 48 individuals employed at St. James's Hospital, Dublin. This is an overall response rate of $32 \%$ based on an approximate overall figure of 326 individuals employed across the two ED's.

At CUH, 51 people were surveyed out of a total of 176 (29\%), while at SJH, 48 out of 150 people were surveyed (32\%). Participation by occupation was as follows: nurses $35 / 111$ (32\%), physicians $34 / 85$ (40\%), physiotherapists $2 / 12$ (17\%), phlebotomists $1 / 4$ (25\%), radiographers $3 / 13$ (23\%), auxiliary staff $20 / 61$ (33\%) (this comprises admin staff $4 / 15(27 \%)$, security $6 / 23(26 \%)$, health care assistants $5 / 12$ $(42 \%)$, porters $5 / 11(45 \%)$ ) and paramedic staff $4 / 40(10 \%)$ ).

Applying the cut-off of 2.18 to the Oldenburg burnout inventory, the percentage of participants testing positive for burnout overall was $74 \%$. The mean level of burnout experienced was 2.42 (standard deviation $=0.37$ ). The subscales of burnout overall showed a mean disengagement level of 2.25 and mean exhaustion level of 2.59. We can again apply the cut-off of 2.18 to these figures. The study demographics are summarised in Table 1.

Our score was normally distributed; we analysed this using a histogram and the Shapiro-Wilk test (SWT). The SWT did not reject the null hypothesis - that the data presented is normally distributed - it did not achieve a level of significance less than $P=0.05(P=0.359)$. Inferring from this, we used parametric testing. Thus, data is presented in Fig. 1 as mean, range and SD across all staff groups.

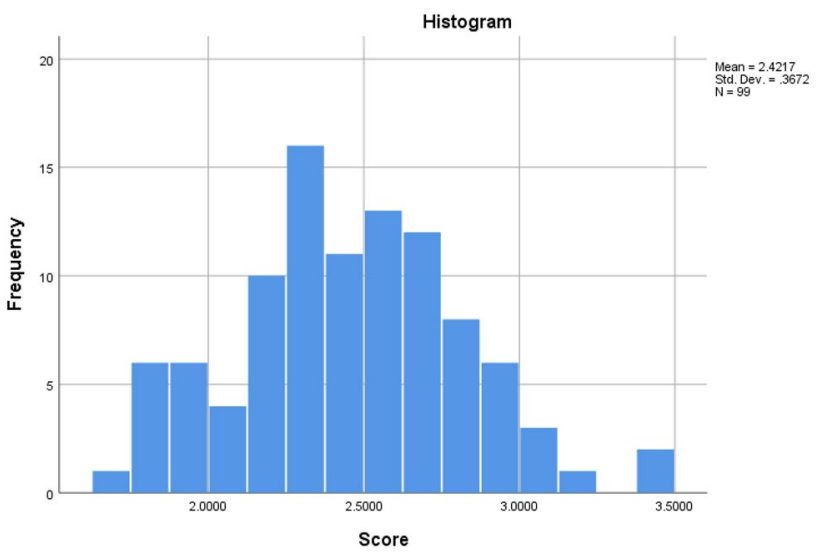

Fig. 1 The $X$ axis represents score in the OLBI, with each 0.1 variance in score grouped together. The $\mathrm{Y}$ axis represents the number of individuals in the bracket shown

\section{Burnout by hospital}

Burnout rates were not significantly different between the hospitals studied $\left(\chi^{2}=2.338, P=0.126\right)$ (based on the Pearson chi-square test). The $t$-test was utilised for burnout scores and was not significant $(t=-0.2, P=0.84)$.

Interestingly, $\mathrm{SJH}$ did show a trend toward higher burnout rates at $81.25 \%(N=48)$ and a higher mean burnout score at 2.42. CUH had a lower burnout rate at $66.6 \%(N=51)$ and a slightly lower mean burnout score at 2.41 . These differences were not statistically significant.

\section{Burnout by occupation}

Burnout rates and scores as experienced by each occupation are summarized in Table 1. Applying a cut-off of 2.18, we found that $77 \%$ of nurses $(N=35 / 111(32 \%)), 76 \%$ of physicians $(N=34 / 85(40 \%)), 50 \%$ of physiotherapists $(N=2 / 12(17 \%)), 100 \%$ of phlebotomists $(N=1 / 4(25 \%))$, $0 \%$ of radiographers $(N=3 / 13(23 \%)), 75 \%$ of paramedics $(N=4 / 40(10 \%))$ and $75 \%$ of auxiliary staff $(N=20 / 61$ (33\%)) met the criteria we had established for burnout. Differences in burnout levels between occupations were not statistically significant. $P$-values (based on the Pearson chi-squared test) for burnout rates and Z-scores (based on

Table 2 No significant difference in burnout levels by occupation

\begin{tabular}{lll}
\hline Occupation: & Z-Scores & Chi-squared \\
\hline Nurses & $T=-1.04 P=0.30$ & $X=0.48 P=0.51$ \\
Physicians & $T=0.48 P=0.63$ & $X=0.20 P=0.66$ \\
Auxiliary staff & $T=-0.95 P=0.346$ & $X=0.00 P=0.99$ \\
Other & $T=2.06 P=0.064$ & $X=3.23 P=0.073$ \\
\hline
\end{tabular}




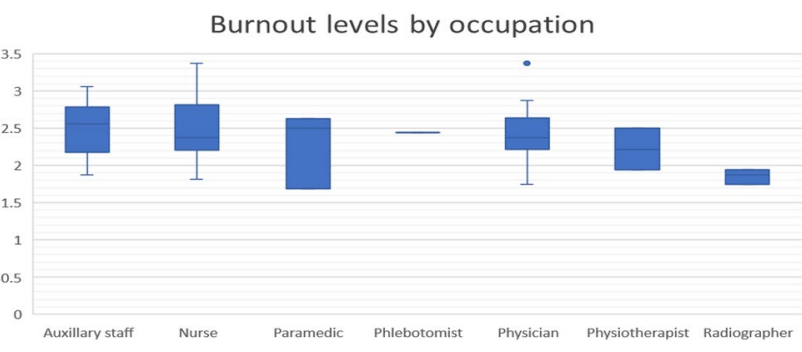

Fig. 2 The variance in burnout by occupation

the student $t$-test) for burnout scores for each occupation are summarised in Table 2.

Due to the small numbers of physiotherapists, radiographers, phlebotomists and paramedics recruited in the study, these populations have been grouped together as "other" in the table.

Figure 2 illustrates a box plot highlighting the distribution of burnout scores by occupation (Fig. 2).

Burnout scores compared against years working in the ED and burnout scores compared against age are demonstrated in Figs. 3 and 4 respectively. We applied the Pearson chisquared test to investigate a relationship between the categorical data (burnout rates) and the student $t$-test to assess continuous data (burnout scores). The variance evident in the burnout rates and scores was not statistically significant for any group in either case $(P=>0.05)$.

\section{Burnout by gender}

This study recruited 39 males and 60 females. These figures reflect approximately the demographic of the emergency department workforce across the departments. The burnout rates experienced between genders were similar: $74 \%$ for females and $73 \%$ for males $(P=0.867)$.

\section{Burnout by history of depression}

There was no clear link between those who experienced burnout and those who self-reported a history of depression.

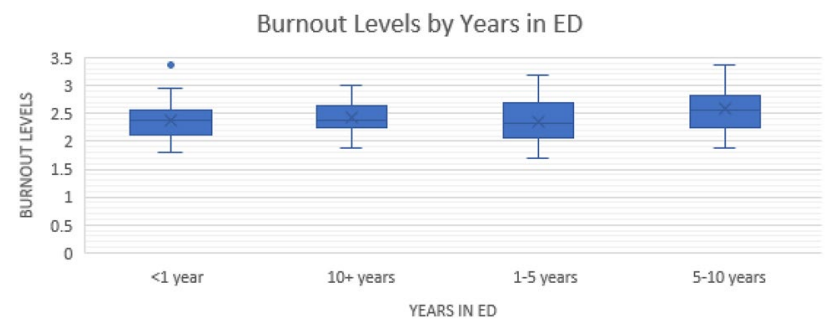

Fig. 3 Burnout level by years in ED

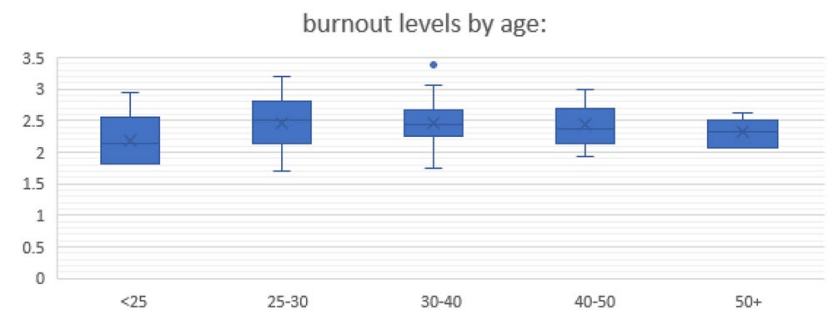

Fig. 4 Burnout levels by age

\section{COVID-19 and staff wellbeing}

Almost all (97\%) of ED staff across the departments had been exposed to patients being investigated for COVID-19 in the department in the previous 6 months.

We included 9 novel questions relating to how healthcare workers felt COVID-19 had impacted on their wellbeing. The results of these questions are summarised in the histogram (Fig. 5).

Increased requirement for personal protective equipment (PPE) was a negative for healthcare workers with $53 \%$ stating it had a negative impact on their wellbeing. Of the remaining $47 \%$, the majority (29\%) viewed PPE as a positive. Requirement for social distancing between staff was again viewed as a negative by most staff, with $54 \%$ stating it had a negative impact on wellbeing. Requirement for social distancing outside of work was also viewed primarily as a negative; $65 \%$ of staff identified this as having a negative impact on wellbeing. Interestingly, decreases in number of visitors attending with patients generated the most agreement with two-thirds (67\%) of staff viewing it as having a positive impact. Other factors identified as positive were, increased prehospital screening and changes to the structure and layout of the emergency department with $55 \%$ and $59 \%$ of staff respectively identifying these as having a positive impact. The remaining factors discussed did not deliver a clear consensus on the opinions of staff.

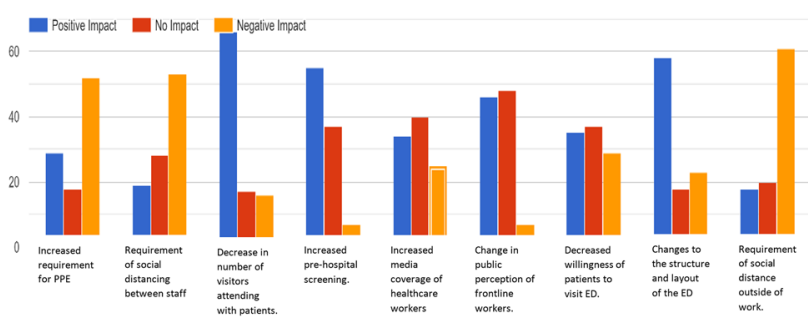

Fig. 5 Nine novel questions relating to how healthcare workers felt COVID-19 had impacted on their wellbeing 


\section{Discussion}

This survey of ED staff working across Ireland's two largest EDs (Cork University Hospital and St. James's Hospital Dublin) aimed to elucidate the degree of workplace well-being across all staff. We measured occupational stress and burnout using a standardised tool (the OLBI). Burnout was found in almost three-quarters of respondents (74\%), indicating that the staff of Ireland's EDs are under substantial occupational strain. The mean exhaustion level (2.59) was higher than any study we are aware of at the time of publication. There was no statistically significant variation between staff by demographics (i.e. occupation, years working in the emergency department, age and gender). However, some trends were recorded; namely, a lower age and fewer years employed in the emergency department were both associated with a decreased level of burnout. There was no significant difference in burnout rates or scores between St. James's Hospital, Dublin, and Cork University Hospital, though the former tended to have a higher rate of burnout at $81 \%$ vs the $67 \%$ burnout observed in CUH.

This study is the first to examine burnout in Irish frontline workers, in the context of the COVID-19 pandemic. Several research papers have been published outside of Ireland addressing the topic. A large study in the UK found the prevalence of burnout to have increased significantly among healthcare staff in the context of the pandemic and reported a burnout rate of $79 \%$ [16]. A similar study in Malaysian frontline workers also saw an increase in burnout at 53\% [22]. On the other hand, the previously mentioned study in Wuhan, China, perhaps the first major study of this kind published, found a marked decrease in burnout amongst staff working on the pandemic's frontline [12]. Although it appears that current international research remains inconclusive, the timing of research needs to be acknowledged so that the phase of the pandemic studied is clear because there may be a unifying effect on staff during the early stages.

Studies investigating burnout levels in Irish ED staff prior to the pandemic show a very high burnout rate. One such study published in 2018 was carried out by Chernoff et al. [15]. This study employed the same instrument to study burnout (the OLBI) and the same cut-off (2.18) as our study [15]. In the 2016 study, $75 \%$ of a cohort of 95 emergency department staff in CUH met criteria for burnout. This is very similar to our overall result (74\%). The mean overall burnout scores were similar in the 2016 study at 2.45 as compared to our finding of 2.42 . Interestingly, there was a trend towards lower burnout levels in our CUH population at $67 \%$, but this was not statistically significant $(P=0.30)$ (determined using Pearson's chi-square test). Mean burnout subscales were also not statistically significant between the research papers, published just 4 years apart, but in different circumstances. The 2016 project found a mean disengagement level of 2.30 and a mean exhaustion level of 2.57 [15]. Our research found a trend towards higher levels of exhaustion at 2.59 (which is the highest reported in any study we are aware of), and lower levels of disengagement from work at 2.25.

Several other pre COVID-19 studies have reported a similar rate of burnout to ours among physicians. An international review undertaken by Arora et al. found a 65\% burnout rate amongst emergency department physicians [6]. By way of comparison, the general population typically experiences a burnout rate in the range of 13-27\% [17]. The large 2019 study previously mentioned [8] used a selfreported "conservative methodology" for its description of burnout. The study found a burnout rate of $29.7 \%$, much lower than that reported in our study (74\%). The studies cannot be directly compared as they used different tools to measure burnout. However, the 2019 study also reported a measure of emotional exhaustion with which comparison to our study may be more suitable (They had employed a different tool which makes direct comparison less meaningful). They reported over $50 \%$ of respondents described high levels of emotional exhaustion, and $82 \%$ were found to have significant levels of occupational stress [8].

A secondary aim of this project was to address how the variety of factors associated with the pandemic, which we included in our survey, have impacted on mental wellbeing. No previous studies have included our additional 9-part questionnaire. The information generated is a novel addition to the literature. We identified some interesting trends in this data. Firstly, an increased requirement for social distancing both between staff and outside of work was associated with a negative impact on staff wellbeing. This was an expected result as social isolation has long been established in the literature as contributing to burnout [4]. Prior to the outbreak, interventions addressing this issue had been outlined and implemented in many workplaces [4]. It stands to reason that these and new protective methods would be important, both during and after the COVID-19 pandemic. Recommendations on how to do this are beyond the scope of this paper. An increased requirement for personal protective equipment was also viewed as having a negative impact on wellbeing by most staff. This suggests that as the pandemic becomes increasingly under control, measures to safely reduce the use of PPE may help to protect the psychological wellbeing of staff.

Interestingly, several of the changes seen in the EDs were associated with a positive impact on staff wellbeing. A decrease in visitors attending the ED with patients was perhaps surprisingly viewed as positive by most staff. This change could potentially be maintained in the post-pandemic world of the future. More research first needs to be carried 
out on this topic, and in particular, an investigation into the impact such a change may have on patients and relatives. Changes to the structure and layout of the ED were deemed a positive change by many staff. These changes were realised as the pandemic saw a considerable influx of capital into healthcare [21]. As a result, an overdue overhaul of ED structure and resources was carried out. This organisational and structural change should, if feasible, be maintained into the future.

Increased media coverage of the healthcare industry, a change in public perception of frontline workers and a decreased willingness of patients to visit the ED were all considered by most staff to have "no impact" on their wellbeing.

\section{Limitations}

A lower participation rate than was expected at $30 \%$ may negatively impact on the power of this study and thereby decrease our ability to confidently interpret results. This study was designed in a cross-sectional manner. This was inevitable given the unpredictability of the pandemic at that time. As a result, a causal link cannot be established between COVID-19 and high rates of staff burnout.

Several potentially relevant factors would have been useful to further the scope of this project. For example, investigating absenteeism, hours worked per week and work life balance. These have all been shown to have a considerable association with burnout [18]. This study includes both burnout scores and burnout rates, but the lack of research in the COVID-19 era employing the OLBI as a measurement tool allows less comparison of scores with international data.

Our study employed a cut-off score of 2.18 to the OLBI; this was chosen to allow for comparison with past studies [14, 15]. 2.18 may be considered excessively low and may be an indicator of occupational stress rather than full-blown burnout (scores can vary from 1 to 4 ). A cut-off score of 2.5 would be more conservative [16]. The OLBI does not state explicitly what the cut-off should be, and previous research argues this point.

Our additional COVID-19-specific questions relating to staff wellbeing were limited. At the time of writing, there was very little international research to support the validity of these questions. As such, the novel contribution to the literature that they provide will be needed to be validated by further research.

\section{Strengths}

In-person invitations to participate may reduce the selfselection bias present in many studies of this kind. Our brief questionnaire proved accessible to staff and was accepted over the course of the shift of a large portion of staff who were invited to participate.

The findings of our study provide an early and timely insight into health care worker burnout in the setting of Irish ED's.

\section{Conclusion}

Towards the end of 2019, a new pandemic emerged worldwide. Ireland's healthcare system was catapulted rapidly into a period of uncertainty and change. The situation evolved quickly, and the consequences for both healthcare workers, and indeed, the general population, are still being felt acutely. For a long time prior to this pandemic, burnout in healthcare staff has been on the rise, and perhaps those worst affected are emergency department staff.

Since the onset of this pandemic, in excess of 18 months ago, there has been continuous strain and seemingly constant "new waves" of exposure for frontline staff. Despite this, COVID-19 has not affected the ED burnout scores reported. It seems the pandemic has offered both positives and negatives to healthcare staff from a wellbeing viewpoint. With that said, this study reiterates what is already known; firstly, that almost three quarters of ED staff are currently experiencing significant levels of burnout in Ireland and secondly that health care workers are exhausted; the mean exhaustion level seen here is higher than any previously recorded to our knowledge at submission.

The trajectory of this disease is still unclear. Consistent, progressive measures to address staff wellbeing, and support frontline workers, are imperative going forward.

\section{Appendix}

See Figs. 6, 7 and 8. 


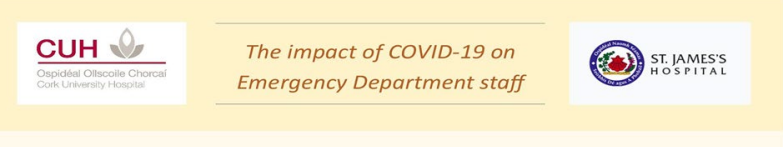

\section{BURNOUT ON THE FRONTLINE}

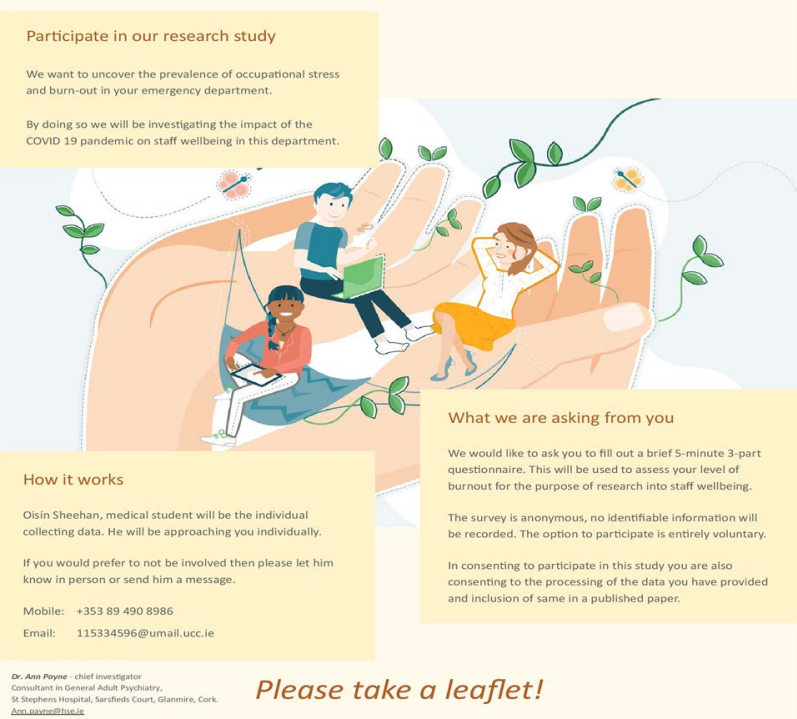

Fig. 6 Information posters were displayed in the EDs of CUH and SJH

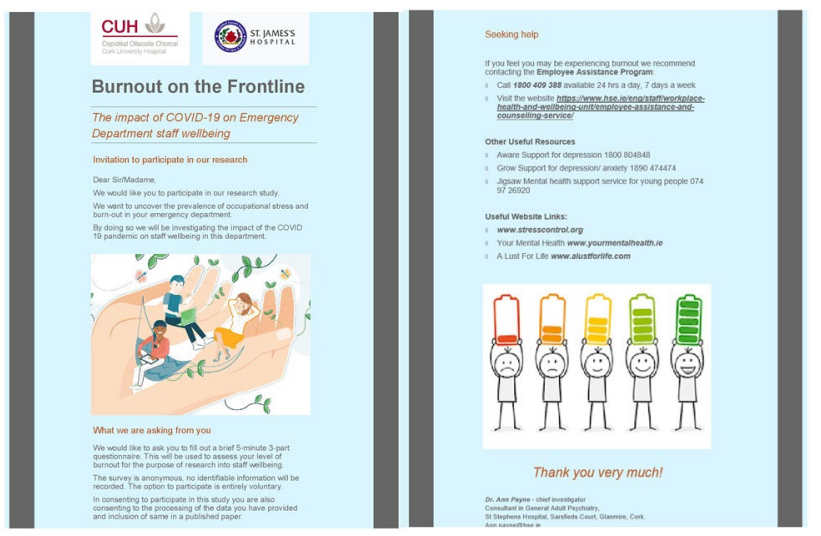

Fig. 7 Brief verbal introduction to the study

\begin{tabular}{|c|c|c|c|c|}
\hline & $\begin{array}{c}\text { Strongly } \\
\text { agree }\end{array}$ & Agree & Disagree & $\begin{array}{l}\text { Strongly } \\
\text { disagree }\end{array}$ \\
\hline I. I always find new and interesting aspects in my work. & 1 & 2 & 3 & 4 \\
\hline 2. There are days when I feel tired before I arrive at work. & $i$ & 2 & 3 & 4 \\
\hline $\begin{array}{l}\text { 3. It happens more and more often that I talk about my work in a negative } \\
\text { way. } \\
\text { 4. After work. I tend to need more time than in the past in order to relax and }\end{array}$ & 1 & 2 & 3 & 4 \\
\hline feel better. & 1 & 2 & 3 & 4 \\
\hline 5. I can tolerate the pressure of my work very well. & 1 & 2 & 3 & 4 \\
\hline 6. Lately, I tend to think less at work and do my job almost mechanically. & 1 & 2 & 3 & 4 \\
\hline 7. I find my work to be a positive challenge. & 1 & 2 & 3 & 4 \\
\hline 8. During my work, I often feel emotionally drained. & i & 2 & 3 & 4 \\
\hline 9. Over time, one can become disconnected from this type of work. & $i$ & 2 & 3 & 4 \\
\hline 10. After working, I have enough energy for my leisure activities. & 1 & 2 & 3 & 4 \\
\hline 11. Sometimes I feel sickened by my work tasks. & i & 2 & 3 & 4 \\
\hline 12. After my work. I usually feel worn out and weary. & 1 & 2 & 3 & 4 \\
\hline 13. This is the only type of work that I can imagine myself doing. & 1 & 2 & 3 & 4 \\
\hline 14. Usually, I can manage the amount of my work well. & 1 & 2 & 3 & 4 \\
\hline 15. I feel more and more engaged in my work. & 1 & 2 & 3 & 4 \\
\hline 16. When I work, I usually feel energized. & 1 & 2 & 3 & 4 \\
\hline
\end{tabular}

14, 16. (R) means reversed item when the scores should be such that higher scores indicate more burnout.

Fig. 8 Burnout levels in staff by utilising the Oldenburg Burnout Inventory (OLBI)

\section{References}

1. Maslach C, SchaufeSli WB, Leiter MP (2001) Job burnout. Annu Rev Psychol 52:397-422. https://doi.org/10.1146/annurev.psych. 52.1.397

2. Shanafelt TD, Boone S, Tan L (2012) Burnout and satisfaction with work-life balance among US physicians relative to the general US population. Arch Intern Med 172(18):1377-1385

3. Shanafelt TD, Hasan O, Dyrbye LN (2015) Changes in burnout and satisfaction with work-life balance in physicians and the general US working population between 2011 and 2014. Mayo Clin Proc. 90(12):1600-1613

4. Adriaenssens J, De Gucht V, Maes S (2015) Determinants and prevalence of burnout in emergency nurses: a systematic review of 25 years of research. Int J Nurs Stud 52(2):649-661

5. Kumar S (2016) Burnout and doctors: prevalence, prevention and intervention. Healthcare 4(3):37 Available from: http://www. mdpi.com/2227-9032/4/3/37

6. Arora M, Asha S, Chinnappa J, Diwan AD (2013) Review article: burnout in emergency medicine physicians. Emerg Med Australas 25(6):491-495 Available from: http://doi.wiley.com/https://doi. org/10.1111/17426723.12135

7. Shanafelt TD, Boone S, Tan L et al (2012) Burnout and satisfaction with work-life balance among US physicians relative to the general US population. Arch Intern Med 172(18):1377-1385

8. Hayes B, Prihodova L, Walsh G et al (2019) Doctors don't Dolittle: a national cross-sectional study of workplace well-being of hospital doctors in Ireland. BMJ Open 9:e025433. https://doi.org/ 10.1136/bmjopen-2018-025433

9. Adams JG, Walls RM (2020) Supporting the health care workforce during the COVID-19 global epidemic. Jama. 323(15):1439-1440 
10. Boyraz G, Legros DN (2020) Coronavirus disease (COVID-19) and traumatic stress: probable risk factors and correlates of posttraumatic stress disorder. J Loss Trauma 1-20

11. Styra R, Hawryluck L, Robinson S (2008) Impact on health care workers employed in high-risk areas during the Toronto SARS outbreak. J psychosomatic Res. 64(2):177-183

12. Wu Y, Wang J, Luo C (2020) A comparison of burnout frequency among oncology physicians and nurses working on the front lines and usual wards during the COVID-19 epidemic in Wuhan. China J pain symptom Manag 60(1):e60-e65. https://doi.org/10.1016/j. jpainsymman.2020.04.008

13. Demerouti E, Mostert K, Bakker AB (2010) Burnout and work engagement: a thorough investigation of the independency of both constructs. J Occup Health Psychol 15(3):209-222. Available from:. https://doi.org/10.1037/a0019408

14. Ruwaard J, Lange A, Schrieken B, Emmelkamp P (2011) Efficacy and effectiveness of online cognitive behavioral treatment: a decade of interapy research. Stud Health Technol Inform 167:9-14

15. Chernoff P, Adedokun C, O'Sullivan I et al (2019) Burnout in the emergency department hospital staff at Cork University Hospital. Ir J Med Sci 188(2):667-674. https://doi.org/10.1007/s11845-0181871-5. (Epub 2018 Jul 26 PMID: 30051165)

16. Ferry AV, Wereski R, Strachan FE et al (2020) Predictors of healthcare worker burnout during the COVID-19 pandemic. medRxiv. https://doi.org/10.1101/2020.08.26.20182378.
17. West CP, Dyrbye LN, Shanafelt TD (2018) Physician burnout: contributors, consequences and solutions. J Intern Med 283(6):516-529. https://doi.org/10.1111/joim.12752. (Epub 2018 Mar 24 PMID: 29505159)

18. Poulsen MG, Poulsen AA, Khan A et al (2015) Recovery experience and burnout in cancer workers in Queensland. Eur J Oncol Nurs 19(1):23-28. Available from: 1016/j.ejon.2014.08.003

19. World Health Organization database figures 06/02/2021. https:// covid19.who.int/

20. World Health Organization (2020) International Statistical Classification of Diseases and Related Health Problems. 11th ed. World Health Organization; Geneva, Switzerland

21. https://www.irishtimes.com/life-and-style/remaking-ireland-fivepositives-from-the-pandemic- 1.4265083

22. Roslan NS, Yusoff MSB, Asrenee AR, Morgan K (2021) Burnout prevalence and its associated factors among Malaysian Healthcare Workers during COVID-19 pandemic: an embedded mixed-method study. Healthcare 9:90. https://doi.org/10.3390/ healthcare 9010090

Publisher's Note Springer Nature remains neutral with regard to jurisdictional claims in published maps and institutional affiliations. 